\title{
A 2-MW Motor And ARCP Drive for High-Speed Flywheel
}

\begin{abstract}
A high-speed induction motor and soft-switching electronic drive are selected to transfer energy between a flywheel and the other power components of prototype hybrid locomotive power system. The motor-generator operates in direct coupling at $14,000 \mathrm{rpm}(233 \mathrm{~Hz}) \mathrm{with}$ the composite flywheel. The motor drive rating is 2 MW (2600 hp) over the operating frequency range of 125 to $250 \mathrm{~Hz}$. The power-frequency range and harmonic heating considerations have lead to a auxiliary resonant commutation pole (ARCP) soft-switching design. The drive and motor have been tested over the full speed range. Unique requirements of energy storage and transfer are also presented.
\end{abstract}

\section{DIGEST}

\section{Introduction - The project}

A hybrid locomotive propulsion system has been developed [1][4] in prototype form using high speed machines, in the 12,000 RPM to 15,000 RPM range, for system size \& mass reduction. The project is directed towards the demonstration of enabling technologies for the incremental implementation of high-speed rail transportation. This paper presents the motor and drive developed for the interface of the flywheel to the turbine-alternator and traction drive systems. Energy transfer is conducted by means of the common DC bus. The energy stored (100 $\mathrm{kWH})$, and power transfer rate (2 MW) place the reported machine and drive technology in the range of interest to small utility, ships' power, and other particularly cyclic power system loads [2][3]. 


\section{Prior Technology}

The flywheel battery (FWB) is a one-terminal energy storage system (like the familiar chemical battery storage system) having a single power connection to the DC bus. Internally, the FWB is composed of the flywheel mass, motor-generator and inverter, and the controls and auxiliaries to have it function to sink, store, and source energy as needed by the larger system. The FWB is compact due to its high rotational speed, but at the cost of a higher operating frequency for its drive motor. A two-pole induction motor design was selected to limit the drive power frequency to $250 \mathrm{~Hz}$ at the flywheel's top speed of 15,000 RPM. By operating at high, direct-drive speeds the motor is physically smaller, however, this feature also affects its thermal capacity. Because of these considerations, a variable speed motor drive was sought with undermodulated PWM output over the full frequency and power range, and a high switching frequency.

\section{The Chosen Topology}

The drive inverter is selected to operate in two quadrants (motoring and generating in one spin direction), and control a resistive load for safe dissipation of flywheel energy. Because it is an energy storage device, the flywheel has a very high rotational moment of inertia (about 389 $\left.\mathrm{kg} \cdot \mathrm{m}^{2}\right)$ as compared to its power rating $(2 \mathrm{MW})$. Therefore, the flywheel energy transfer through the motor is torque-controlled, the power delivered or absorbed being defined by torque at the current speed. The flywheel motor [7] has been designed for a base voltage of 1200 V-RMS with a nominal dc link voltage of $1960 \mathrm{~V}$-DC. This allows full operation within the linear range of the amplitude modulation index $\left(m_{a}<1.0\right)$, avoiding harmonic voltage distortions due to overmodulation. 
A switching frequency of $4 \mathrm{kHz}$ was chosen to exceed 15 times the power frequency over the full operating range. The switching frequency, while fixed, can be changed during operation to optimize the suppression of further harmonic distortion due to unfavorable frequency modulation ratios $\left(m_{f}\right)$. In order to conduct the required current at this switching frequency, it was determined to use a soft-switching topology, specifically, the auxiliary resonant commutation pole (ARCP) topology of quasi-resonant swtiching [5]. As such, this drive system would be among the largest soft-switching motor drives built to date [6].

A load resistor circuit is included to provide an energy dump for the flywheel upon shutdown. The resistor switch is designed both as a hard-switch circuit, using hysterisis switching for over voltage and voltage balance control, but also with a $500 \mathrm{~Hz}$ PWM switching for variable load (on demand) control.

The ARCP design uses a switched auxiliary inductor leg on each motor phase leg to cause a resonance with the capacitance across the main switch. This resonant circuit brings the voltage across the switch to zero before closing. Thus, the auxiliary (inductor) switch is turned on at will, having a limited turn-on di/dt, and is controlled to turn off at the following current zero; and the main inverter switches are turned on at voltage zero and turned off at will, having the $\mathrm{dv} / \mathrm{dt}$ controlled by directly connected capacitors.

The ARCP drive relies on the switch rhythm to be in play, so the initial conditions do not allow the first main switch closure. Some provision must be made to short the first main switch to operate in each of the three phase legs. To accomplish this, a smaller IGBT circuit was built, with a series resistance, to discharge the lower phase leg switch capacitors just before an initial gate signal is allowed to complete. In this way the normal sensor control becomes valid, and detects the required zero voltage at the switch before allowing the gate signal. 


\section{The Controller}

Within the motor drive proper it was decided to leverage a commercial drive control board to provide the basic six-gate switching sequence and motor feedback. The switching signals are captured in a field programmable gate array (FPGA) which has been programmed to transform the six-switch sequence to the nine-switch requirements in the ARCP configuration. The output of the FPGA is conveyed by optical fibers to the gate drive boards, which gate each IGBT in a switch-group ( 3 parallel by 2 series) after a verified voltage zero is detected across the switch. The FPGA also handles the drive initiation logic to operate the discharge switch in anticipation of the initial phase switching events.

The drive is equipped with a water cooling system and uninteruptible control power. These ancillary components are started and diagnosed in sequence by a power converter supervisory control. This controller also interfaces with external power demands, translating them to flywheel torque commands, and feeds status and alarms up to the power system operator.

\section{Testing \& Results}

The initial VFD build was completed in November of 2003, but was transferred to our laboratory after early test failures for continued development and testing. After a period of circuit study and tests, signal handling logic and some hardware was revised. The inverter has since been tested with inductors and the flywheel's induction motor-generator as loads. Figure 1 shows the FW motor-generator and the inverter photo is seen in Figure 2. Loss analysis for the inverter and the motor show the contributions of current harmonics and those due to other causes. 


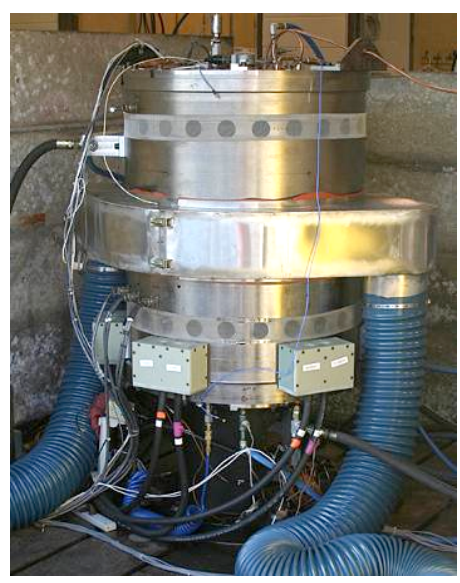

Figure 1. Motor-Generator

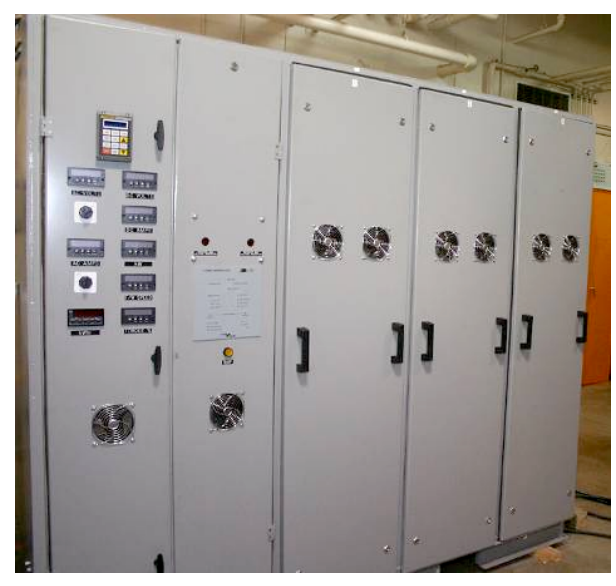

Figure 2. Inverter 